\section{Paradoxos das políticas de descentralização de saúde no Brasil}

\author{
Dário Frederico Pasche, ${ }^{1}$ \\ Liane Beatriz Righi, ${ }^{1}$ \\ Henrique Inácio Thomé ${ }^{1}$ \\ e Eveline Dischkaln Stolz ${ }^{1}$
}

A constituição federal do Brasil (1), no artigo 198, estabelece que as ações e serviços públicos de saúde integram uma rede regionalizada e hierarquizada, constituindo um sistema único, organizado de acordo com as diretrizes da descentralização, do atendimento integral e da participação social. Os processos de descentralização podem ser classificados como desconcentração, devolução e delegação (2). A desconcentração sugere a transmissão de certas responsabilidades e funções, sem a transferência correspondente de poder decisório. A delegação é a transferência de responsabilidades gerenciais para organismos não-governamentais. Por sua vez, a devolução implica na transferência de poder decisório para as esferas subnacionais, fortalecendo-as.

No que se refere ao Brasil, a classificação que melhor se aplica ao Sistema Único de Saúde (SUS) seria a de descentralização como devolução. Descentralização, nessa perspectiva, implica na ampliação da autonomia das esferas estaduais e municipais, que requisitarão qualificação para a realização de um conjunto de funções públicas.

A implementação dessa diretriz constitucional, ao longo dos últimos 15 anos, tem tomado o sentido da processualidade que decorre da própria conjuntura do campo sanitário, bem como dos contextos políticos desenhados nas disputas político-partidárias. Portanto, buscar compreender o processo de descentralização e a conseqüente ampliação das responsabilidades das esferas subnacionais, sobretudo nos anos 1990, quando efetivamente essa estratégia ganhou terreno, requer uma investigação histórica, exigindo a reconstrução de cenários políticos e a identificação de sujeitos e da direcionalidade de suas ações e projetos.

Este artigo pretende reconstituir o processo de devolução de responsabilidades no âmbito da saúde às esferas subnacionais no Brasil. $\mathrm{O}$ período compreendido nesta análise abarca os anos de 1993 a 2004. Esses limites coincidem com a emergência da Norma Operacional Básica (NOB) 01/93 do SUS (3), que recoloca a questão da descentralização da saúde, e com o fim do mandato do Ministro da Saúde Humberto Costa, do partido governista, o Partido dos Trabalhadores (PT), agremiação reconhecida por sua ação política de aposta e apoio ao SUS.

\section{O CONTEXTO POLÍTICO DO BRASIL NOS ANOS 1990}

Nos anos 1990, consolidou-se a hegemonia das políticas neoliberais, levadas a cabo, na maioria dos países, por meio de uma agenda denominada de 
ajuste estrutural (4). No Brasil, foram consolidadas propostas de redução e restrição das atividades de bem-estar, com precarização das relações de trabalho, geração de superávit primário, abertura da economia para empresas transnacionais, privatização de empresas estatais e redefinição do escopo das funções do Estado, situando-as, predominantemente, na regulação, controle e avaliação, entre outros (5-7).

Embora haja um grande dissenso quanto ao impacto dessa agenda na área da saúde no Brasil, é possível dizer que suas proposições centrais foram "amortecidas" devido, de um lado, à inscrição jurídico-legal do SUS, sobretudo às concepções de direito à saúde e participação cidadã; e, de outro lado, ao processo de descentralização de serviços e ações, que, nos anos 1990, tomou forte impulso, ampliando a presença da esfera municipal e o leque de sujeitos sociais que, em alguma medida, passaram a resistir à "onda neoliberal" (8).

A agenda neoliberal produziu forte influência nas políticas públicas brasileiras. No campo da saúde, pode-se perceber um duplo movimento: ataque à prodigalidade do sistema público de saúde, notadamente sua universalidade; e resistência política e institucional, com ressignificação de agendas, a partir do qual proposições como descentralização e focalização foram desdobradas em estratégias de consolidação do sistema público e universal de saúde.

Além disso, o financiamento da saúde -elemento de grande interesse da agenda de ajustes- passou, nos anos 1990, por um conjunto de redefinições, cujo desfecho foi a aprovação, em 2000, da emenda constitucional 29 (EC/29). Esta emenda estabelece que os estados passem a investir $12 \%$ da receita de impostos estaduais em serviços e ações de saúde; os municípios, $15 \%$ da receita de impostos municipais; e a União, o montante aplicado no ano anterior corrigido pela variação nominal do produto interno bruto (PIB). Em 2001, o gasto público em saúde no Brasil atingiu $3,2 \%$ do PIB. Embora haja divergências quanto ao impacto da emenda (se resultou ou não na ampliação do gasto em saúde), a introdução desse tipo de mecanismo constitucional tende a limitar a ação restritiva dos governos no gasto em saúde, constituindo-se em um avanço importante frente às ameaças ${ }^{2}$ impostas pelos governos neoliberais.

\section{A era Collor}

Os anos 1990, no Brasil, sob a perspectiva política, foram paradoxais. O processo de transição

\footnotetext{
2 Em comunicação pessoal (2003), Nelson Rodrigues dos Santos (que foi secretário-executivo do Conselho Nacional de Saúde) relatou que uma das imposições feitas por Stanley Fischer, chefe de algumas missões do Fundo Monetário Internacional (FMI) no Brasil, era a ação rápida do governo Fernando Henrique Cardoso para impedir a aprovação da emenda constitucional 29. Uma outra recomendação, não menos ofensiva, seria acabar com os soviets, os conselhos de saúde.
}

democrática se consolidou pela eleição do governo liberal e conservador de Fernando Collor de Mello, que deu início à onda neoliberal no país. Na área da saúde, esse governo produziu um "boicote sistemático e contundente à implantação do SUS" (9), que pode ser comprovado tanto pela aprovação com vetos da lei 8 080/90 (10), o que na prática impossibilitava a participação cidadã e a transferência automática de recursos para as esferas subnacionais $(11,12)$, bem como pelos sucessivos adiamentos da IX Conferência Nacional de Saúde, realizada, finalmente, em 9 de agosto de 1992.

O governo de Fernando Collor teve um efeito de retrocesso na área da saúde. Tal retrocesso é evidenciado pela diminuição dos recursos (13) e pela edição, ainda pelo Instituto Nacional de Assistência Médica da Previdência Social (INAMPS), da NOB 01/1991 (14), que consolidou o município como um mero prestador de serviços, com pouca autonomia para organizar redes de atenção à saúde em seu território. Já no final do governo Collor (fevereiro de 1992), denúncias de corrupção promoveram a saída do Ministro Alceni Guerra e a entrada de Adib Jatene. Essa mudança não repercutiu em uma nova direção para a área da saúde, nem incrementou os gastos públicos no setor (9). A condução da política de saúde nesse período foi muito parecida com aquela verificada antes da criação do SUS.

Frente à perspectiva de cassação de seu mandato, em 1992 o presidente Collor renunciou. Tomou posse, então, o vice-presidente Itamar Franco, que promoveu uma série de iniciativas importantes no campo da saúde, entre as quais merece destaque a extinção do INAMPS em 1993 (15). Por 15 anos, o INAMPS coordenou a política de centralização administrativa e financeira do sistema de saúde, responsável pela consolidação do modelo de atenção à saúde privatista e essencialmente médico. A força e a marca da ação político-institucional desse instituto ficaram conhecidas como "cultura inampsiana" (16).

Na gestão do ministro Jamil Haddad, verificouse um esforço para definir o papel de cada esfera de governo, bem como para ampliar os recursos destinados à saúde mediante destinação de percentuais mínimos para a área em cada esfera de governo. Além disso, buscou-se, num quadro recessivo, ampliar a receita, racionalizar os custos da assistência e erradicar o desperdício (13). Ainda nessa gestão ocorreu um deslocamento importante na direção do processo de descentralização, com a edição da NOB 01/1993 (3), que aponta para a construção de um processo que leva em consideração a autonomia dos municípios, os quais poderiam, a partir de determinadas capacidades e responsabilidades, assumir a gestão do sistema de saúde, exercendo um conjunto de macrofunções de gestão.

A edição dessa norma está circunscrita ao cenário de efervescência do movimento municipalista brasileiro (17). Através da IX Conferência Nacional 
de Saúde, convocada sob a insígnia "municipalização é o caminho" e subsidiada pelo documento "Descentralização das ações e serviços de saúde: a ousadia de cumprir e fazer cumprir a lei" (18), houve enorme pressão para que o SUS fosse efetivamente implantado, sugerindo a ampliação da participação dos municípios no processo de descentralização. O início dos anos 1990 foi, portanto, importante para a consolidação do SUS, sobretudo pelo fomento ao processo de municipalização, marca maior da diretriz constitucional da descentralização político-administrativa.

Ao mesmo tempo, paradoxalmente, esse período foi também marcado por forte desfinanciamento do setor (8), com grande oscilação do volume de recursos. Isso determinou, entre outros, o congelamento das tabelas de remuneração do SUS e a ausência de expansão dos investimentos, ocasionando o sucateamento da rede pública e a queda na qualidade da atenção, a ampliação das filas de espera, a sub-remuneração dos profissionais, etc.

\section{A era FHC}

Em 1995 assumiu um novo governo, encabeçado pelo ex-ministro da fazenda do governo Itamar Franco, o sociólogo e senador Fernando Henrique Cardoso (FHC), que obteve um segundo mandato de 1999 a 2002 com esmagadora vitória. $\mathrm{O}$ governo $\mathrm{FHC}$ deu continuidade à agenda neoliberal (19), cujas expressões mais pungentes podem ser visualizadas no processo agudo de privatização de empresas estatais e na construção de uma nova institucionalidade para o Estado brasileiro.

$\mathrm{Na}$ área da saúde, o primeiro ministro foi Adib Jatene, que permaneceu no cargo por 2 anos. Esse período foi marcado pela luta pessoal do ministro com a área econômica por mais recursos para a saúde, feito obtido mediante a instituição do Imposto Provisório sobre Movimentação Financeira (IPMF), hoje Contribuição Provisória sobre a Movimentação ou Transmissão de Valores e de Créditos e Direitos de Natureza Financeira (CPMF). Além disso, na gestão de Jatene foi realizada a $X$ Conferência Nacional de Saúde e aprovada a NOB 01/96 (20).

Entre a gestão de Adib Jatene e a de José Serra, a partir de março de 1998, não ocorreu efetivamente nenhuma mudança no cenário políticoinstitucional, com exceção de algumas inovações introduzidas pela NOB 01/96, sobretudo o estímulo à implantação dos Programas de Agentes Comunitários de Saúde e Saúde da Família (PACS e PSF, respectivamente).

A edição de uma nova NOB nesse período reafirma o caminho e a disposição de se implantar o SUS por meio de atos normativos do Ministério, "esculpindo o SUS a golpes de portarias" (21), não se observando qualquer movimento no sentido da regulamentação das leis orgânicas da saúde. Com a NOB 01/96 retomou-se o tom tecnocrático, que mais tarde foi realçado pela Norma Operacional da Assistência à Saúde (NOAS) (22).

Mesmo tendo em vista que os atos normativos ministeriais resultam de pactuação na Comissão Intergestores Tripartite (CIT), composta por três representantes de cada uma das esferas de governo (o Ministério da Saúde, o Conselho Nacional de Secretários Estaduais de Saúde, ou Conass, e o Conselho Nacional de Secretários Municipais de Saúde, ou Conasems), que se reúnem ordinariamente uma vez ao mês para discutir e aprovar normas, estratégias e projetos da área da saúde, em todo o período a expressão "furor normativo" passou a ser utilizada para descrever o processo de burocratização implementado pelo executivo federal, que, no final dos anos 1990, editava em média oito portarias por dia útil.

Esse comportamento institucional revela que ainda era marcante a presença da cultura inampsiana (16) no interior do Ministério da Saúde. A orientação normativa pautou a ação do governo que, à época, concordava com as análises dos organismos multilaterais de crédito de que o principal problema da saúde no País decorria da baixa capacidade de gestão das esferas subnacionais, o que elevava os índices de ineficiência. Nessa perspectiva, cabia ampliar o processo normativo, diminuindo o grau de governabilidade dos gestores sobre os recursos que deveriam ser gerenciados por meio de "caixinhas" — uma expressão utilizada por especialistas em financiamento do SUS para designar a grande quantidade de mecanismos de repasse de recursos aos gestores subnacionais, que atualmente supera a casa dos 300.

No que se refere às políticas de saúde, o governo FHC seguiu, em linhas gerais, duas grandes pautas: de um lado, fomentou políticas estratégicas conduzidas diretamente por seus ministros, sobretudo Serra, a exemplo dos medicamentos genéricos, da saúde da família, da política de combate à AIDS e da regulação da medicina suplementar (já que o sistema de saúde brasileiro é, na verdade, um sistema plural, composto pelo sub-sistema público, ou SUS, o sub-sistema de medicina suplementar, ou seguros, as cooperativas médicas, os sistemas de administração, entre outros, e o desembolso direto); de outro lado, ampliou o processo de reconcentração de competências regulatórias sobre as ações assistenciais no Ministério da Saúde. Assim, nesse período, houve uma re-centralização aguda de comando no gestor federal, obtida por meio do arrefecimento da ação política da CIT e do estreitamento dos canais de diálogo com o Conselho Nacional de Saúde $(8,9)$.

Também nesse período, sob a perspectiva da ação programática, se exerceu forte ação de fomento ao Programa de Saúde da Família, que passou a ser uma das principais ações do Ministério da Saúde. 
As 787 equipes de saúde da família que existiam em 1995 foram ampliadas para 16847 em 2002 (23). Além disso, ocorreram em 1998 a implementação do piso de assistência à saúde ${ }^{3}$, que passou a destinar recursos per capita para a atenção básica, uma antiga reivindicação do movimento sanitário registrada na lei 8080 (10), e a ampliação da modalidade de repasse fundo-a-fundo. Essas ações ampliaram a autonomia dos municípios e estados e diminuíram drasticamente os contratos e convênios, mecanismos com potencial para favorecer o clientelismo.

Sob a perspectiva normativa deve-se destacar a edição da NOAS, que, entre outros, conferiu ao processo de descentralização novas perspectivas, ampliando-o para além da municipalização da saúde, que se fortaleceu através da regionalização da oferta de recursos assistenciais. A oferta de instrumentos de gestão regional, notadamente o Plano Diretor de Regionalização, cuja construção considera, entre outros, a programação integrada e pactuada, passou a estimular a construção de processos de programação regional, acionando, em certa medida, o exercício de funções gestoras, que se diferenciam daquelas de gerência, conforme havia sido definido pela NOB 01/96.

A regionalização, a partir de então, passou a ser considerada um mecanismo importante para se ampliar o acesso eqüitativo ao SUS, permitindo, entre outros, a criação e a ampliação de redes de atenção regional. Contudo, as diversidades regionais e a desigualdade de capacidades e acúmulos técnicos entre equipes locais e secretarias estaduais de saúde, coordenadoras do processo, determinaram um processo de regionalização bastante heterogêneo, com avanços significativos em algumas regiões e manutenção de redes fragmentadas em outras (2).

\section{A era Lula}

Em 2003 assume o governo Lula, com a promessa de aliar o desenvolvimento econômico e a ampliação de políticas de proteção social. O Programa Fome Zero (24) foi a expressão mais contundente da proposta desse governo, pois articulou um conjunto de ações governamentais e não-governamentais, promovendo a distribuição de alimentos a partir do fortalecimento da agricultura familiar e estimulando a formação de cooperativas, a criação de infra-estrutura, a geração de empregos formais e o acesso à educação nutricional. Entretanto, o programa causou polêmica no interior do próprio governo, envolvendo membros do Ministério da Fazenda, ministros e dirigentes da área social, sobre o aporte de novos recursos para a saúde, educação, cultura, desporto, entre outros.

3 Estabelecido pela NOB 01/96, sua denominação foi alterada a partir da NOAS 01/2001 para Piso de Atenção à Saúde.
Instalou-se, dessa forma, já no início do governo, um embate sobre a orientação macroeconômica, considerada continuista (25), e a necessidade de ampliar os gastos sociais. O desfecho desse embate aponta para o fortalecimento das orientações da área econômica (26). O governo optou, sobretudo, por ampliar os mecanismos de transferência de recursos diretos às famílias, por meio do programa Bolsa Família. Esse programa se destina a famílias com renda per capita de até R\$ 100 mensais (27). São utilizados recursos de várias pastas, inclusive da saúde. O programa Bolsa Família reúne um conjunto de benefícios anteriormente dispersos (vale-gás, bolsa-escola, etc.), condicionando-os a responsabilidades assumidas pelas famílias, como manter as crianças em idade escolar na escola.

O jogo político entre as áreas econômica e social determinou que o quadro de financiamento do SUS fosse mantido inalterado, com a União investindo recursos federais em conformidade ao estabelecido pela EC/29, muito embora tenha recebido críticas por debitar aos recursos do SUS gastos não considerados como ações e serviços de saúde (26). Assim, paradoxalmente, o fluxo regular de recursos tem correspondido a incrementos no volume do gasto em saúde a cada ano, sem, contudo, determinar aumento no percentual do PIB investido em saúde $^{4}(8)$. Desse volume de recursos, aproximadamente $90 \%$ são transferidos diretamente aos estados e municípios, através das transferências fundoa-fundo. Entretanto, continua sendo criticada a forma fragmentada dos repasses, que reduz a autonomia dos dirigentes da saúde na gestão dos recursos de acordo com seus planos de saúde, como estabelecido por lei (10).

A equipe que assumiu o Ministério da Saúde em janeiro de 2003, sob coordenação do ministro Humberto Costa e do secretário-executivo Gastão Wagner de Sousa Campos, passou a reunir lideranças partidárias, sobretudo do PT, e sanitárias, ligadas à tradição acadêmica, bem como ex-gestores estaduais e municipais do SUS. Ao assumir, a equipe produziu uma reforma administrativa que ampliou a possibilidade de integração entre diversas áreas (28). A secretaria executiva passou a ter papel de destaque, promovendo uma ação política de transversalidade.

A política de descentralização entrou na agenda sob o tema do "fortalecimento da gestão descentralizada", o que determinou o incremento na relação entre gestores das três esferas de governo, com reestruturação da CIT e de sua pauta. Foram constituídos, junto à secretaria técnica, sete grupos de trabalho, e a dinâmica da pauta de discussões passou a destacar quatro pontos: análise de

\footnotetext{
4 O gasto com saúde em 1995 correspondeu a 14,5\% da arrecadação federal; em 2001, com a vigência dos cálculos da EC/29, recuou para $11,4 \%(8)$.
} 
pleitos de habilitação; temas para discussão; pactuação de políticas; e informes gerais. Também foram reconfiguradas as relações político-institucionais entre Conass, Conasems e o Ministério da Saúde, coordenadas desde então pelo Departamento de Apoio à Descentralização (DAD). Esse processo de fortalecimento da CIT impôs uma nova dinâmica à produção e implementação de políticas de saúde, na medida em que a aprovação de novos projetos e estratégias passou a estar condicionada a consensos produzidos nessa comissão, respaldada por discussões de sua câmara técnica e grupos temáticos. Além disso, o Ministério passou a qualificar a gestão descentralizada, introduzindo inovações como o apoio integrado (29) e a Rede de Cooperação Técnica e Apoio à Gestão do SUS (CooperaSUS) (30).

Entretanto, do ponto de vista das relações formais entre as esferas de governo, pouco se avançou. Em todo o período, em que pesem os esforços do grupo de trabalho tripartite para aprovar um novo pacto de gestão (31), não se definiram novas regras para a descentralização, continuando em vigor a NOAS 01/2002 (22), que recebeu alguns reparos mínimos na CIT. O tema da regionalização, ponto importante para um novo processo de contratualização de responsabilidades sanitárias, embora tenha sido bastante discutido entre as partes, não se efetivou como um lugar importante de mudança.

Nos 2 primeiros anos do governo Lula, o Ministério da Saúde adotou uma série de medidas incrementais, como a correção do repasse do piso de atenção básica (PAB), o aumento dos valores financeiros das equipes de saúde da família e o estímulo à implantação de novas equipes (23) incluindo as de saúde bucal. No ano de 2003 foram implantadas 2,4 mil novas equipes, que passaram a somar 20000 em todo o País, cobrindo 80 milhões de brasileiros. O PSF estava presente, em fevereiro de 2005, em 4590 municípios brasileiros, com 21391 equipes que atendiam cerca de 69 milhões de pessoas (39\% da população do país).

Além dessas medidas, houve uma série de incrementos nos gastos com a atenção de média e alta complexidade, corrigindo-se preços fixados em tabelas, além da inclusão de novos procedimentos. Uma medida importante foi a volta da equiparação da tabela para serviços públicos e privados, que na gestão Serra haviam sido diferenciados, com valores majorados apenas para os serviços privados. Foram implantadas novas equipes no sistema nacional de transplantes e credenciados novos serviços; criados novos leitos para terapia intensiva; ampliados os repasses para assistência farmacêutica, tanto básica como de medicamentos de alto custo e para o tratamento do HIV / AIDS; foram criados novos centros de atenção psicossocial, que se constituem em estratégia de desospitalização psiquiátrica.
Outras políticas e iniciativas importantes foram adotadas nesse período, entre as quais cabe destacar a elaboração do Plano Nacional de Saúde; o Programa Farmácia Popular; a política nacional de humanização da atenção e gestão dos serviços de saúde; a criação do Serviço Médico de Urgência (SAMU); a política de educação permanente do SUS; o início das discussões em torno da avaliação de desempenho do SUS; e a elaboração do plano nacional diretor de investimentos.

Embora a implementação dessas medidas sugira uma maior presença da esfera pública na composição do gasto em saúde, dados de 2003 (32) revelam que apenas $44 \%$ dos recursos eram provenientes do setor público, enquanto $56 \%$ correspondiam a gastos realizados por indivíduos e famílias, que obtiveram assistência médica através de desembolso direto ou através de seguros e planos de saúde privados (33).

A escolha do usuário pela assistência médica suplementar, ou seja, a opção pelo pagamento para a obtenção de serviços médicos, é mediada e fomentada por mecanismos de renúncia fiscal e estímulos publicitários. Na prática isso implica em abrir mão do direito ao acesso gratuito, conforme determina a base jurídico-legal do SUS. Embora essa situação esteja presente no SUS, que em alguma medida mantém mecanismos que a reproduzem, ela emergiu em meados da década de 1960, quando se verificou estímulo público à criação de serviços privados (34), fenômeno que determinou um curioso quadro denominado de "universalização excludente". Essa denominação expressa a paradoxal situação de ampliação do acesso aos serviços de saúde concomitantemente ao estímulo à saída de segmentos da classe média, que passou a se afastar, de forma geral, da política pública de saúde.

Em que pesem essas considerações, a forte presença do gasto privado (de famílias) com saúde revela uma situação de iniqüidade e descompasso entre a base discursiva e a real efetivação da política pública de saúde no Brasil. Em um país que elege a saúde com um bem de relevância pública, a constatação de que o maior gasto tem origem privada sinaliza que há ainda um longo caminho a ser percorrido no reconhecimento da saúde como pleno e inalienável direito de cidadania.

Todo esse período, entretanto, foi marcado por dificuldades de se levar adiante as discussões em torno do pacto de gestão, sobretudo os mecanismos de transferência financeira, a regionalização e a responsabilização sanitária, o que revela a existência de divergências na condução política pelo Ministério da Saúde no período de 2003 e 2004, tendo como clímax a saída do secretário-executivo, em novembro de 2004, 8 meses antes da queda do ministro, substituído por um representante da base aliada do presidente Lula. 


\section{CONSIDERAÇÕES FINAIS}

É inequívoca a ampliação da presença de esferas subnacionais na gestão e na provisão de serviços de saúde. Essa participação começa de forma discreta nos primeiros anos da década de 1980, com as ações integradas de saúde, e se amplia ao longo de toda a década, em cujo terço final as secretarias estaduais, por meio dos Sistemas Unificados e Integrados de Saúde (SUDS) (35), expandem sua participação político-institucional. Mas é, sobretudo, a partir da edição da NOB 01/93 (3) que os municípios consolidam sua presença no SUS, no marco da descentralização entendida como devolução (2).

Tal participação, contudo, pode ser chamada de paradoxal, pois uma maior presença das esferas subnacionais na gestão do SUS foi levada a cabo mediante perda relativa de autonomia para desenvolver em seus territórios redes e estratégias de atenção à saúde. Isso se deveu, sobretudo, à presença e à ação de resquícios da cultura inampsiana (16) no interior do Ministério da Saúde, que passou a utilizar critérios excessivamente normativos para o regramento da presença das esferas subnacionais na gestão do SUS. A reversão dessa situação impõe a introdução de novos mecanismos e medidas que sejam capazes de desfazer esse equívoco voluntário sobre o processo de descentralização, que o tem tornado, em muitas circunstâncias, processo de desconcentração.

É certo, contudo, que, nos últimos anos, muitos avanços foram implementados no sistema público de saúde, ainda que, em sua grande maioria, em uma linha incrementalista (com exceção de alguns, como a política de educação permanente, o programa de urgências e emergências, a política nacional de humanização da atenção e da gestão, entre outros). A aproximação do SUS real ao SUS inscrito na legislação, entretanto, além de medidas incrementais, necessita de iniciativas que possam corrigir distorções e contradições, a exemplo dos mecanismos de repasse de recursos, e qualificar os processos de gestão. Isso exige, necessariamente, um novo pacto de gestão, capaz de ampliar o grau de responsabilidades macro e micro-sanitárias entre gestores, sobretudo nos espaços local e estadual, que juntos, com o apoio do Ministério da Saúde, devem construir as múltiplas conexões no âmbito regional.

Em agosto de 2005 a CIT voltou a pautar as discussões referentes à produção de um novo acordo político envolvendo as três esferas de governo, propondo pactos em defesa do SUS, da vida e de gestão. Esses temas compõem uma "agenda de compromisso pela saúde" (36), cuja elaboração e aprovação pela CIT (37) indica que há em curso, no SUS, um processo político que poderá reafirmar esse sistema como política de Estado.

Um novo processo de pactuação, entretanto, deve caminhar no sentido de ampliar a autonomia decisória da esfera municipal. Para isso são necessários o apoio e a cooperação técnica e financeira das secretarias estaduais de saúde e do Ministério, que devem ampliar suas formas de apoio, deslocando a predominância da supervisão e controle, herdados da cultura inampsiana (16). A estratégia do apoio integrado (29) é uma aposta nessa direção, e se constitui em inovação na gestão da política pública de saúde brasileira.

Um movimento de tal envergadura exige e impõe como condição a produção de um novo pacto sanitário nacional, que deve envolver, além de gestores, parlamentares, organismos da sociedade civil e a população em geral. A introdução de inovações nas formas de organização e de gestão do sistema de saúde brasileiro compõe uma complexa agenda política, que dialoga com muitos interesses de vários setores sociais. Nessa medida, o alargamento dos espaços de debate, a ampliação da participação da sociedade e a transparência no processo de discussão é condição sine qua non para a consolidação de um sistema público e democrático. A qualificação e o fortalecimento da descentralização da saúde no Brasil impõem como desafio a produção de novas relações federativas, afirmativas da atuação com autonomia sem, contudo, abdicar da ação cooperativa e solidária.

\section{SYNOPSIS}

\section{Paradoxes of health decentralization policies in Brazil}

The constitution of Brazil directs that the country's health system, the Unified Health System (Sistema Unico de Saúde), be politically and administratively decentralized. Nevertheless, handing over competencies, responsibilities, and resources to subnational levels, especially to municipal governments, has been a slow process, lasting almost two decades. Advances have been brought about by the Unified Health System, which, from a analytical perspective, is a public and universal system. Despite that, the decentralization process needs to overcome norms that keep all levels of management dependent on Brazil's federal Government. The subnational levels have consistently faced difficulties in performing their macromanagement functions with autonomy, especially when it comes to financing and to the establishment or organization of health care networks. Boldness and responsibility will be needed to prevent Brazil's health decentralization process from leading to fragmentation. New political agreements between different levels of government, with a reassignment of responsibilities and the enhancement of a culture of technical cooperation, are fundamental requisites to making the Unified Health System have a health policy that is truly public and universal.

Key words: delivery of health care; health care reform; models, organizational; public health administration; social responsibility; Brazil. 


\section{REFERÊNCIAS}

1. Brasil. Constituição Federal de 1988. Diário Oficial da União 1988. 5 de outubro:1 (anexos, col. 1).

2. Mendes EV. Os grandes dilemas do SUS: tomo II. Salvador: Casa da Qualidade Editora; 2001.

3. Brasil, Ministério da Saúde. Portaria MS No 545, 20 de maio de 1993 Brasil. Diário Oficial da União 1993. 24 de maio.

4. Pasche DF. Gestão e subjetividade em saúde [tese de doutorado]. Campinas: UNICAMP, Faculdade de Ciências Médicas, Departamento de Medicina Preventiva e Social; 2003.

5. Ugá MAD. Ajuste estrutural, governabilidade e democracia. Em: Gerschman S, Vianna MLW, orgs. A miragem da pósmodernidade: democracia e políticas sociais no contexto da globalização. Rio de Janeiro: Fiocruz; 1997. Pp. 81-100.

6. Antunes R. Os sentidos do trabalho: ensaio sobre a afirmação e a negação do trabalho. $2^{a}$ ed. São Paulo: Boitempo; 2000.

7. Forrester V. Uma estranha ditadura. São Paulo: UNESP; 2001.

8. Barros E. Saúde: idas e vindas do financiamento da política de saúde. Em: Rocha D, Bernardo M, orgs. A era FHC e o governo Lula: transição? Brasília: Instituto de Estudos Socioeconômicos; 2004. Pp. 151-90.

9. Bravo MIS, Matos MC. A saúde no Brasil: reforma sanitária e ofensiva neoliberal. Em: Bravo MIS, Pereira PAP, orgs. Política social e democracia. São Paulo: Cortez; 2001. Pp. 197-216.

10. Brasil, Ministério da Saúde. Lei $N^{\circ} 8080$ (Lei Orgânica da Saúde). Dispõe sobre as condições para a promoção, proteção e recuperação da saúde, a organização e o funcionamento dos serviços correspondentes e dá outras providências. Diário Oficial da União 1990. 20 de setembro:18055 (col. 1)

11. Arretche M. A política da política de saúde no Brasil. Em: Lima NT, Gerschman S, Edler FC, Suárez JM, orgs. Saúde e democracia: história e perspectivas do SUS. Rio de Janeiro: Fiocruz; 2005. Pp. 285-306.

12. Cordeiro H. Descentralização, universalidade e eqüidade nas reformas da saúde. Cienc Saude Coletiva. 2001;6(2): $319-28$.

13. Gerschman S. A democracia inconclusa: um estudo da reforma sanitária brasileira. Rio de Janeiro: Fiocruz; 1995.

14. Brasil, Ministério da Previdência e Assistência Social. Resolução Inamps N $^{\circ}$ 258, de 07 de janeiro de 1991. Aprova a Norma Operacional Básica $\mathrm{N}^{\circ}$ 01/ 91. Diário Oficial da União 1991. 10 de janeiro.
15. Brasil, Presidência da República, Casa Civil. Lei $N^{\circ} 8689$ de 27 de julho de 1993. Dispõe sobre a extinção do Instituto Nacional de Assistência Médica da Previdência Social (Inamps) e dá outras providências. Diário Oficial da União 1993. 28 de julho.

16. Mendes EV. Uma agenda para a saúde. São Paulo: Hucitec; 1996.

17. Carvalho G. A inconstitucional administração pós-constitucional do SUS através de normas operacionais. Cienc Saude Coletiva. 2001;6(2):435-44.

18. Brasil, Ministério da Saúde, Grupo Especial de Descentralização. Municipalização das ações de saúde. A ousadia de cumprir e fazer cumprir a lei. Brasília: Ministério da Saúde; 1993.

19. Sader E, Gentili P, orgs. Pós-neoliberalismo: as políticas sociais e o Estado democrático. São Paulo: Paz e Terra, 1995.

20. Brasil, Ministério da Saúde. Portaria MS $\mathrm{N}^{\circ} 2$ 203, de 5 de novembro de 1996. Aprova a Norma Operacional Básica do Sistema Unico de Saúde, NOB-SUS 01/96. Diário Oficial da União 1996. 6 de novembro.

21. Goulart FAA. Esculpindo o SUS a golpes de portaria ... —considerações sobre o processo de formulação das NOBs. Cienc Saude Coletiva. 2001;6(2):292-8.

22. Brasil, Ministério da Saúde. Portaria ${ }^{\circ}$ 373/GM, de 27 de fevereiro de 2002. Diário Oficial da União 2002. 28 de fevereiro:52 (seção 1).

23. Brasil, Ministério da Saúde. Cobertura da estratégia da saúde da família-2002. Disponível em: http://dtr2002.saude. gov.br/caadab / 2002/Tabela $\% 201 \% 20$ $\% 20$ Cobertura \%202002.pdf. Acessado em dezembro de 2005

24. Brasil, Presidência da República. Fome Zero. Disponível em: http://www.fome zero.gov.br/. Acessado em dezembro de 2005.

25. Sader E. A vingança da história. São Paulo: Boitempo; 2003.

26. Associação Brasileira de Pós-graduação em Saúde Coletiva (Abrasco), Centro Brasileiro de Estudos de Saúde (Cebes), Associação Brasileira de Economia da Saúde (Abres), Rede Unida, Associação Nacional do Ministério Público em Defesa da saúde (Ampasa). Reforma sanitária brasileira - manifesto: reafirmando compromissos com a saúde brasileira. Brasília: 2005. Disponível em: http:// www.abrasco.org.br/Congresso $\% 20$ Saude $\% 20$ Coletiva $\% 202006 /$ REFORMA $\% 20$ SANIT\%C1RIA \%20BRASILEIRA $23 \% 20$ novembro\%20.pdf. Acessado em dezembro de 2005.

27. Brasil, Ministério do Desenvolvimento Social e Combate à Fome. Bolsa Família.
Disponível em: http://www.mds.gov. br/bolsafamilia/bolsafamilia01.asp. Acessado em dezembro de 2005.

28. Barros E. Brasil: o perfil do sistema de serviços de saúde. Disponível em http://www.opas.org.br/servico/temas documento_detalhe.cfm?CodSubTema= $70 \&$ CodEspecifico $=5515$. Acessado em dezembro de 2005

29. Brasil, Ministério da Saúde. Apoio integrado à gestão descentralizada do SUS. Apoiador: experiências partilhadas, soluções coletivas. Brasília: Ministério da Saúde/Organização Pan-Americana da Saúde; 2005

30. Brasil, Ministério da Saúde, Departamento de apoio à descentralização, Rede CooperaSUS. Disponível em: www. saude.gov.br/cooperasus. Acessado em dezembro de 2005

31. Brasil, Ministério da Saúde, Departamento de Apoio à Descentralização. Documentos de subsídio para o pacto de gestão. Disponível em: http://dtr2001. saude.gov.br/dad/. Acessado em dezembro de 2005

32. Ugá MA, Santos IS. Uma análise da eqüidade do financiamento do sistema de saúde brasileiro [relatório de pesquisa]. Rio de Janeiro: ENSP/FIOCRUZ 2005.

33. Dain $\mathrm{S}$, Faveret AC, Cavalcanti CE, Carvalho D, Cuadros WL. Avaliação dos impactos de reforma tributária sobre o financiamento da saúde. Em: Negri B, Giovanni GD, orgs. Brasil: radiografia da saúde. Campinas, SP: Unicamp; 2001. Pp. 233-88.

34. Oliveira JAA, Fleury S. (Im)Previdência social: 60 anos de história de previdência no Brasil. Petrópolis: Vozes; 1989.

35. Levcovitz E, Noronha JC. AIS-SUDSSUS: os caminhos do direito à saúde. Em: Guimarães R, Tavares RAW, orgs. Saúde e sociedade no Brasil: anos 80. Rio de Janeiro: Relume Dumará; 1994; Pp. 73-111.

36. Brasil, Ministério da Saúde, Conselho Nacional dos Secretários de Saúde, Conselho Nacional de Secretarias Municipais de Saúde, Comissão Intergestores Triparte (CIT). Resumo executivo-reunião 18 de agosto de 2005. Disponível em: http://dtr2001.saude.gov.br/dad/ tripartite/Resumos/Res_Ago_05.pdf. Acessado em janeiro de 2006.

37. Brasil, Ministério da Saúde, Departamento de Apoio à Descentralização, Comissão Intergestores Tripartite. Calendário 2006. Resumo executivo da reunião do dia 26 de janeiro de 2006. Disponível em: http://dtr2001.saude.gov.br/ dad/tripartite/Resumos/Res_Jan_06. pdf. Acessado em janeiro de 2006. 